OITS 694

July 2000

\title{
Observable measures of critical behavior in high-energy nuclear collisions
}

\author{
Rudolph C. Hwa \\ Institute of Theoretical Science and Department of Physics \\ University of Oregon, Eugene, OR 97403-5203, USA
}

\begin{abstract}
Critical behaviors of quark-hadron phase transition in high-energy heavy-ion collisions are investigated with the aim of identifying hadronic observables. The surface of the plasma cylinder is mapped onto a $2 \mathrm{D}$ lattice. The Ising model is used to simulate configurations corresponding to cross-over transitions in accordance to the findings of QCD lattice gauge theory. Hadrons are formed in clusters of all sizes. Various measures are examined to quantify the fluctuations of the cluster sizes and of the voids among the clusters. The canonical power-law behaviors near the critical temperature are found for appropriately chosen measures. Since the temperature is not directly observable, attention is given to the problem of finding observable measures. It is demonstrated that for the measures considered the dependence on the final-state randomization is weak. Thus the critical behavior of the measures proposed is likely to survive the scattering effect of the hadron gas in the final state.
\end{abstract}

\section{Introduction}

The subject of this talk can be introduced by commenting on the four conceptual items mentioned in the title, but in reverse order. First, high-energy nuclear collisions. The goal is to create quark-gluon plasma. So far the conventional signatures are aimed at detecting the primordial quark matter. Suppose that is found. Then what? If we can learn from the condensed-matter physics, then we know that critical phenomena form a vibrant area of research. That leads to the second item in the title: critical behavior. Our concern will be the quark-to-hadron phase transition (PT). The hadron-to-quark PT is, in my view, too turbulent in heavy-ion collisions to lend itself to systematic studies, despite some recent attempt to use percolation as a model to characterize it. The third item in the title is "measures." For a thermal system the heat capacity can be a measure that exhibits the critical behavior $C \propto\left|T-T_{c}\right|^{-\alpha}$, while for a magnetic system the magnetic susceptibility behaves as $\chi \propto\left|T-T_{c}\right|^{-\gamma}$. What is the measure for quark-hadron PT? Is there a measure, call it $\mu$, which behaves as $\mu \sim\left|T-T_{c}\right|^{-\kappa}$ ? If so, how do we find it? That brings us to the last item: observable. Temperature is not directly observable. (The transverse momentum $p_{T}$ is an unreliable, gross indicator of the average phenomenon.) Moreover, the system is 
not static and the emitted hadrons may undergo final-state interactions that can smear the signature that is to be observed. The aim of this talk is to show how all these issues are addressed and to indicate where the answers are to be found.

The measure that I believe will convey the information about quark-hadron PT is about fluctuations, not only bin-to-bin fluctuations, but also event-to-event fluctuations. A way to illustrate this belief is to consider the annual precipitation (AP) of two places with widely different climatic characteristics. The AP of certain regions in Oregon and Florida are very similar, about $40^{\prime \prime}$ per year, but their rainfall patterns are nearly opposite: drizzly in Oregon, stormy in Florida. The problem is that in determining the PA the daily rainfall is integrated over the entire year, and then averaged over all years. That is exactly how the average multiplicity of heavy-ion collisions is determined: integrated over the whole hadronization time of an event (which can take over $30 \mathrm{fm} / \mathrm{c}$ ), and then averaged over all events. Clearly, to learn about the nature of rainfall or the properties of hadronization, it is necessary to study spatial and temporal fluctuations.

\section{The Problem}

If quark-gluon plasma is indeed formed in a heavy-ion collision, it is reasonable to assume that the plasma occupies a cylindrical volume, expanding rapidly in the longitudinal direction, and more slowly radially. The medium most likely has a temperature profile that is high at the center of the transverse plane and decreases with increasing radius due to the radial expansion and cooling. When $T$ reaches $T_{c}$ at the surface, the quark matter undergoes a $P T$ into hadrons. Thus the characteristics of critical behavior are to be found on the cylindrical surface at any given time of the evolutionary history of the system. That is like raining at a particular instant of observation. If we collect all the hadrons emitted throughout the long hadronization processes, the features of critical behavior are likely to overlap and get averaged out. It is therefore important to select a narrow $\Delta t$ cut in the hadronization time. If the emission time and the transverse momentum $p_{T}$ of the hadrons are correlated in a oneto-one relationship, then a narrow $\Delta p_{T}$ cut would accomplish what we need. Unfortunately, that relationship is not one-to-one. On the contrary, at each emission time the emitted particles have a very wide $p_{T}$ distribution. That wide distribution turns out to provide us with a way to proceed. Suppose we make a narrow $\Delta p_{T}$ cut and study the hadronic patterns in the $\eta-\phi 2 \mathrm{D}$ space orthogonal to $\vec{p}_{T}$. Then at any emission time only a small fraction of the emitted hadrons would enter the narrow $\Delta p_{T}$ window. This random selection process at each time frame thus decorrelates the hadrons collected over the entire hadronization process. The measured hadronic patterns in the $\eta-\phi$ space is then the superposition of small parts of many configurations (which we call configuration mixing), each of which we shall stimulate before mixing. The object of our analysis is to find the scaling property of each configuration that exhibits the critical behavior, and then to see whether it survives the mixing process.

It is well known that a system at the critical point forms clusters of all sizes that exhibit Kadanoff scaling. The details of the dynamical system are not important, since the critical behavior depends mainly on the dimension and symmetry of the system. On the basis of that universality we shall use the Ising model as a simple device to simulate the configuration 
of hadronic clusters formed on the surface of the plasma cylinder at each time frame of hadronization. There are reasons to believe that the QCD dynamics for the chiral PT suggests a cross-over near the second-order PT that belongs to the same universality class as the Ising system [1]. We shall define hadron formation on the Ising lattice in such a way as to be able to simulate a cross-over. From the simulated configurations we can see the hadronic clusters randomly scattered over a background of voids. In our first-attempt analysis of the patterns formed, we shall ignore the complications that arise from the $p_{T}$ distribution and consider directly the scaling properties of the clusters and voids in uncorrelated configurations that we shall simulate.

\section{Cluster Analysis}

The use of the Ising model on a 2D lattice to simulate second-order PT is standard [2], 3]. To have a cross-over in quark-hadron PT without the use of an external field in the Ising Hamiltonian is not standard [4]. The method uses a cell on the lattice, of dimension $\epsilon \times \epsilon$ where $\epsilon$ may be taken to be 4 , to define hadron density $\rho_{c}$ at the $c$ th cell

$$
\rho_{c}=\lambda \phi_{c}^{2} \theta\left(\phi_{c}\right)
$$

where

$$
\phi_{c}=\sum_{j \in c} \operatorname{sgn}\left(m_{L}\right) \sigma_{j}, \quad m_{L}=\sum_{j \in L^{2}} \sigma_{j} .
$$

The first sum in (目) is over all sites in a cell having site-spins $\sigma_{j}$; the second sum is over all lattice sites, giving the total magnetization $m_{L}$. We use the direction of $m_{L}$ of each configuration to serve as the direction of an external field and define the order parameter $\phi_{c}$ as the cell spin at $c$ relative to that direction. $\lambda$ in (四) is a parameter relating hadron density to Ising spins; all measurable quantities should be insensitive to $\lambda$. Because of the $\theta$-function in (1), $\rho_{c}$ is either positive where hadrons are emitted, or zero where a void exists.

There are two quantities we have found that can effectively serve as measures of fluctuations of hadronic clusters from bin to bin and from configuration to configuration. One is $J(M, T)$ defined

$$
J(M, T)=\left\langle\frac{\rho_{k}}{\langle\rho\rangle} \ln \frac{\rho_{k}}{\langle\rho\rangle}\right\rangle
$$

and the other is $K(M, T)$ whose definition is omitted here, but can be found in Ref. [5]. In (3) $\langle\cdots\rangle$ refers to averaging over all bins $\left[M\right.$ of them, $\left.M=(L / \delta)^{2}\right]$ and over all configurations. We discuss only the properties of $J(M, T)$ here; those of $K(M, T)$ are similar.

What we have found about $J(M, T)$ is that it is factorizable [5], i.e.,

$$
J(M, T)=\alpha(T) J_{c}(M)
$$

where $J_{c}(M)=J\left(M, T_{c}\right)$. Thus, by definition, $\alpha\left(T_{c}\right)=1$. Yet, when $T$ is away from $T_{c}$, but not too far in $T<T_{c}, \alpha(T)$ satisfies the power-law behavior

$$
\alpha(T) \propto\left(T_{c}-T\right)^{-\zeta}, \quad \zeta=1.88
$$


This may be regarded as the critical behavior that we have been searching for. However, $T$ is not directly measurable. We can use $\bar{\rho}$ instead, where $\bar{\rho}$ is the cell density averaged over all cells in one configuration. Then we find

$$
J(M, \bar{\rho})=\alpha(\bar{\rho}) J_{0}(M)
$$

with

$$
\alpha(\bar{\rho}) \propto\left(\bar{\rho}-\bar{\rho}_{0}\right)^{-\bar{\zeta}}, \quad \bar{\zeta}=0.97
$$

where $\bar{\rho}_{0}$ is the average density where $J\left(M, \bar{\rho}_{0}\right)=J_{0}(M)$ is the highest. The behavior in $(\bar{\pi})$ is measurable and can be used to signal the fluctuation property of a quark-hadron PT.

\section{Void Analysis}

In addition to finding the critical behavior of the hadronic clusters, we can also analyze the scaling properties of the voids where no hadrons appear [4. First, it is necessary to define precisely what a void is, and how to avoid a single hadron from changing its quantification. To that end we define a bin to be empty if the average density $\bar{\rho}_{k}$ of the $k$ th bin is less than $\rho_{0}$, where $\rho_{0}$ is a parameter that can be varied by the analyst of the data. We do not set $\rho_{0}$ to zero in order to eliminate the small fluctuations due to irregular occurrences of hadrons in or at the edges of a bin. A void is then the area of contiguous empty bins, and its area is therefore

$$
V_{n}=\sum_{k} \theta\left(\rho_{0}-\bar{\rho}_{k}\right)
$$

where the sum is over all connected empty-bins of the $n$th void. $V_{n}$ is in the unit of number of bins. A quantity less dependent on the size of the bins is the fraction $x_{n}=V_{n} / M$. For every configuration, there exists a set of void fractions $\left\{x_{1}, x_{2}, x_{3}, \ldots x_{m}\right\}$ where $m$ is the total number of voids. The comparison of void patterns of different configurations would be difficult if they are characterized by those sets. To ease that comparison, we define the $G$ moments

$$
G_{q}=\frac{\left\langle x^{q}\right\rangle}{\langle x\rangle^{q}}
$$

where $\left\langle x^{q}\right\rangle=\left(\sum_{n=1}^{m} x_{n}^{q}\right) / m$, an average performed for each configuration. Thus $G_{q}$ is a number (for each $q$ ) that characterizes the void pattern of a configuration. At the critical point this $G_{q}$ fluctuates widely from configuration to configuration. It depends on the bin size.

At $T$ in the vicinity of $T_{c}$ we find that $\left\langle G_{q}\right\rangle$, averaged over all configurations, satisfies scaling law for a variety of $\rho_{0}$ values, i.e.,

$$
\left\langle G_{q}\right\rangle \propto M^{\gamma_{q}}
$$

That means voids of all sizes occur. Furthermore, the scaling exponents depend on $q$ linearly

$$
\gamma_{q}=c_{0}+c q
$$

with $c=0.8$ at $T_{c}$ and $\rho_{0}=20$. This value of $\rho_{0}$ is less than $8 \%$ of the maximum $\bar{\rho}$. The value of $c$ depends on $T$ and $\rho_{0}$ mildly, and can be used to quantify the nature of void patterns. 
Since $G_{q}$ fluctuates very widely, i.e., the distribution $P\left(G_{q}\right)$ is very broad, the moments of $G_{q}$ can reveal that fluctuation. We have studied the derivative of the first moment, which is

$$
S_{q}=\left\langle G_{q} \ell n G_{q}\right\rangle
$$

and found that it also satisfies a scaling law

$$
S_{q} \propto M^{\sigma_{q}}
$$

whose exponent depends on $q$ linearly

$$
\sigma_{q}=s_{0}+s q
$$

with $s=0.76$ at $T=T_{c}$ and $\rho_{0}=20$. The value of $s$ also depends on $T$ and $\rho_{0}$ mildly, and therefore can further be used to quantify the nature of the fluctuation of void patterns from configuration to configuration.

The dependences of $c$ and $s$ on $\rho_{0}$ and $T$, though mild, can be used to diagnose the temperature range of quark-hadron $P T$. For $\rho_{0}>20$ the values of $c$ and $s$ determined in our analysis vary in a range as much as $20 \%$ for $T$ varying in the range of $\pm 2 \%$ around $T_{c}$, but they converge to the values quoted as $\rho_{0} \rightarrow 20$. If $c$ and $s$ are not unique for a range of $T$ around $T_{c}$, it means that $\gamma_{q}$ and $\sigma_{q}$ are not fixed (for fixed $q$ ) if PT takes place over a range of $T$, not exactly at $T_{c}$. Then there would be no scaling behavior. Thus, if the experimental data reveal the loss of scaling as $\rho_{0}$ is increased, it implies that the $P T$ takes place over a range of $T$. If, however, scaling persists, then $P T$ occurs only at one $T$, presumably $T_{c}$.

\section{Complications in Hadronization}

There are two aspects about hadronization in heavy-ion collisions that one should be concerned about for the type of measures we are proposing. One is the effect due to final-state interaction as the hadrons traverse the hadron gas. The other is the effect of configuration mixing. The former is studied by requiring the produced hadron in each configuration to take $\nu$ steps of random walk, since scattering by a hadron gas implies the random deflections of the initial $\vec{p}_{T}$ that result in random wandering in the $\eta-\phi$ plane. We have studied the effect of such random walks (for $\nu$ up to 6 ) on $J(M, T)$ and found that the factorizability (四) persists and that $\alpha(T)$ retains the essential character of (5) [5].

The other complication of configuration mixing due to the non-correspondence between $\Delta p_{T}$ and $\Delta t$ is more difficult to treat. We have considered only the simple case of no correlation between successive time frames. We mix four independently simulated configurations by taking one quadrant from each and then apply our void analysis to the mixed configu-

rations [4]. We find that the effects on $\gamma_{q}$ and $\sigma_{q}$ are essentially negligible. This problem will be followed by a more realistic treatment of the configuration mixing where the wide $p_{T}$ distribution of the emitted particles at any given time will be taken into account together with the hadron-void correlation in successive time frames. 


\section{Multifragmants}

Finally, let me make some remarks about "Common Aspects" that is a part of the title of this session. Despite the valiant attempts made by the organizers of this conference to promote interaction between the two subfields encompassed here, cross-fertilization has not been a vibrant phenomenon that has taken place. However, it seems that the ideas about the void analysis discussed here can be translated into the study of multifragmentation in the intermediate mass range. Let me therefore venture a suggestion on an analysis of the fragments with the hope that it may yield a feature of the experimental results more challenging to the model builders than what they have hitherto dealt with.

Suppose that for each event an experiment can measure a certain number of fragments whose total mass may be less than the total mass of the nuclei in collision and may fluctuate from event to event. Nevertheless, for all the fragments $M_{i}$ that have been detected, one can calculate the fraction $x_{i}=M_{i} / \sum_{i} M_{i}$. If it is the charges of the fragments that are measured, then let $x_{i}=Z_{i} / \sum_{i} Z_{i}$. Each event is then characterized by a set of fractions $\left\{x_{1}, x_{2}, x_{3}, \ldots x_{n}\right\}$. The idea is to go beyond studying the averages and examine the nature of the fluctuation of the fragments detected. Let us define

$$
H_{q}(n)=\frac{1}{n} \sum_{i=1}^{n} x_{i}^{q}
$$

where $n$ is the total number of fragments measure in an event. Clearly, $H_{0}=1$ and $H_{1}=1 / n$. At higher $q, H_{q}(n)$ are progressively smaller, but are increasingly more dominated by the large $x_{i}$ components.

If $P\left(H_{q}\right)$ is the distribution of $H_{q}$ after all events are sampled, then the average is

$$
\left\langle H_{q}\right\rangle=\frac{1}{\mathcal{N}} \sum_{e=1}^{\mathcal{N}} H_{q}^{e}(n)=\int d H_{q} H_{q} P\left(H_{q}\right)
$$

where $e$ labels an event and $\mathcal{N}$ is the total number of events. $\left\langle H_{q}\right\rangle$ provides some average information about the multifragments, but to study the fluctuation from event to event one should calculate the normalized moments of moments [6]

$$
C_{p, q}=\left\langle H_{q}^{p}\right\rangle /\left\langle H_{q}\right\rangle^{p}
$$

This $C_{p, q}$ may contain too much information. The derivative at $p=1$ may already be sufficient to provide some interesting insight into the erratic nature of the fluctuation

$$
E_{q}=\left.\frac{d}{d p} C_{p, q}\right|_{p=1}=\left\langle\frac{H_{q}}{\left\langle H_{q}\right\rangle} \ln \frac{H_{q}}{\left\langle H_{q}\right\rangle}\right\rangle,
$$

the study of which may be called erraticity [7], for convenience. The experimental determination of $E_{q}$ as a function of $q$ may pose a serious challenge to the theoretical models, some of which may not contain enough dynamical fluctuations to generate the erraticity revealed by the data. 


\section{Acknowledgments}

The work described here was carried out at different stages in collaboration with Z. Cao, Y. F. Wu and Q. H. Zhang. It was supported, in part, by the U. S. Department of Energy under Grant No. DE-FG03-96ER40972.

\section{References}

[1] K. Rajagopal, in Quark-Gluon Plasma 2, edited by R. C. Hwa, (World Scientific, Singapore, 1995).

[2] J. J. Binney, M. J. Dowrick, A. J. Fisher, and M. E. J. Newman, The Theory of Critical Phenomena (Claredon Press, Oxford, 1992).

[3] Z. Cao, Y. Gao and R. C. Hwa, Z. Phys. C 72, 661 (1996), Z. Cao and R. C. Hwa, Phys. Rev. E 56, 326 (1997).

[4] R. C. Hwa, and Q. H. Zhang, Phys. Rev. C (to be published), nuch-th/0003032.

[5] R. C. Hwa, and Y. F. Wu, Phys. Rev. C 60, 054904 (1999).

[6] Z. Cao and R. C. Hwa, loc. cit, and Phys. Rev. D 54, 6674 (1996).

[7] R. C. Hwa, Acta Physica Polonica B27, 1789 (1996). 